\title{
Molecular Basis for the Adaptive Evolution of Environment Sensing by H-NS Proteins
}

Xiaochuan Zhao,a Vladlena Kharchenko,b Umar F. Shahul Hameed,c Chenyi Liao,a Franceline Huser,c Jacob M. Remington,c Anand K. Radhakrishnan,c Mariusz Jaremko,b Łukasz Jaremko,b* Stefan T. Arold, $, \mathrm{d}, \mathrm{d}^{*}$ Jianing Lia*

a. Department of Chemistry, The University of Vermont, Burlington, VT, USA 05405.

b. King Abdullah University of Science and Technology (KAUST), Biological and Environmental Science and Engineering (BESE), Thuwal, 23955-6900, Saudi Arabia

c. King Abdullah University of Science and Technology (KAUST), Computational Bioscience Research Center (CBRC), Biological and Environmental Science and Engineering (BESE), Thuwal, 23955-6900, Saudi Arabia

d. Centre de Biochimie Structurale, CNRS, INSERM, Université de Montpellier, 34090 Montpellier, France

Correspondence to: JL (jianing.li@uvm.edu), STA (stefan.arold@kaust.edu.sa) or LJ (lukasz.jaremko@kaust.edu.sa) 
Abstract: The DNA-binding protein $\mathrm{H}-\mathrm{NS}$ is a pleiotropic gene regulator in gram-negative bacteria. Through its capacity to sense temperature and other environmental factors, $\mathrm{H}$-NS allows pathogens like Salmonella to adapt their gene expression, and hence toxicity and biological responses, to their presence inside or outside warm-blooded hosts. To investigate how this sensing mechanism may have evolved to fit different bacterial lifestyles, we compared $\mathrm{H}-\mathrm{NS}$ orthologs from bacteria that infect humans, plants, and insects, and from bacteria that live on a deep-sea hypothermal vent. The combination of biophysical characterization, high-resolution proton-less NMR spectroscopy and molecular simulations revealed, at an atomistic level, how the same general mechanism was adapted to specific habitats and lifestyles. In particular, we demonstrate how environment-sensing characteristics arise from specifically positioned intra- or intermolecular electrostatic interactions. Our integrative approach clarified the mechanism for $\mathrm{H}$ NS-mediated environmental sensing and suggests that it resulted from the exaptation of an ancestral protein feature.

Key words: evolution, adaptation, MD simulations, proton-less NMR, low-gamma detection, autoinhibition. 


\section{INTRODUCTION}

The histone-like nucleoid-structuring (H-NS) protein is a central controller of the gene regulatory networks in enterobacteria (1). H-NS inhibits gene transcription by coating and/or condensing DNA; an environment-sensing mechanism allows $\mathrm{H}-\mathrm{NS}$ to liberate these DNA regions for gene expression in response to physicochemical changes (2-4). H-NS preferentially binds to AT-rich sequences, which enables its dual role in (i) the organization of the bacterial chromosome and (ii) the silencing of horizontally acquired foreign DNAs (5-8). The latter mechanism allows bacteria to assimilate foreign DNAs, which, however, are only expressed as a last resort in case of acute threats or stresses (8). Thus, H-NS plays a crucial role in the adaptation, survivability, and antibiotic resistance of bacteria. Given the growing threat of multidrug resistance, H-NS has attracted increasing research interest, with a particular focus on elucidating the molecular mechanisms of adaptive evolution (9-11).

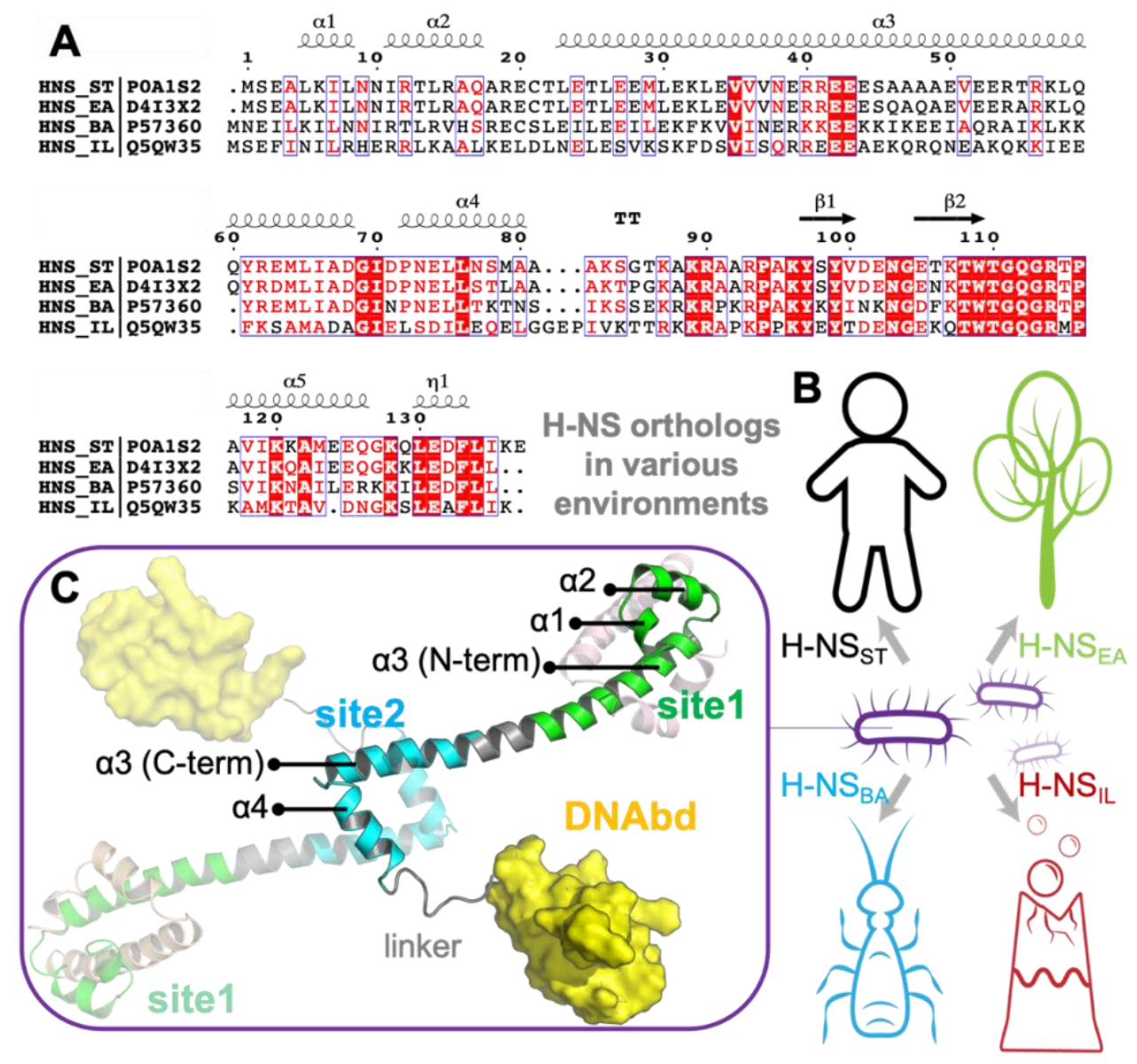

Figure 1. The sequence, structure, and habitat of selected $\mathrm{H}-\mathrm{NS}$ orthologs. (A) Sequence alignment of $\mathrm{H}$ NS orthologs was performed with ESPript 3.0 (12): H-NSst (UniprotID: P0A1S2), H-NSEA (UniprotID: D4I3X2), H-NSBA (UniprotID: P57360), and H-NSIL (UniprotID: Q5QW35). (B) Illustration of the environment of the selected orthologs. (C) The tetrameric H-NSst model we used for MD is shown with each domain 
labelled: site1 (green helices), site2 (cyan helices), linker (grey loop), and DNA-binding domain (yellow surface). The truncated site1 of flanking site1-dimerized chains are shown in magenta and pale orange. The tetramer H-NSst model was shown in cartoon, with each domain labelled: site1 (residues, 1-44), site2 (52-82), linker (83-92), and DNA-binding domain (93-137).

H NS possesses two dimerization domains (site1, residues 1-44; site2, resides 52-82; the numbering of Salmonella typhimurium is adopted throughout the text), and a C-terminal DNAbinding domain (DNAbd, residues 93-137) that is connected through a flexible region (linker, residues 83-92) to site2 (Fig. 1) (5, 13-16). The combination of site1 and site2 dimers allows $\mathrm{H}$ NS to form multimers for a stable concerted DNA association that results in gene silencing (15). In a previous study, we showed that site 2 of $S$. typhimurium $\mathrm{H}-\mathrm{NS}$ is the primary response element to temperature changes (17). Site2 unfolds at human body temperature, allowing the linkerDNAbd region to associate with site 1 to adapt an autoinhibited conformation incapable of binding to DNA. Salinity and pH can also influence the stability of site2 dimers, and hence may also affect gene repression by $\mathrm{H}-\mathrm{NS}(17,18)$. Thus, the sensitivity of $\mathrm{H}-\mathrm{NS}$ to temperature and other physiochemical changes allows human pathogens such as S. typhimurium, Vibrio cholerae, and enterohaemorrhagic Escherichia coli to sense when they enter a homothermic host and adapt their gene expression profiles accordingly.

To date, studies to elucidate environment sensing of $\mathrm{H}-\mathrm{NS}$ were almost exclusively conducted with proteins from two model systems, S. typhimurium (e.g. (9, 19-21)) and E. coli (e.g. $(18,22-26))$, both of which infect humans. Yet, H-NS orthologs are also present in enterobacteria that do not have warm-blooded hosts, raising the question of what biological role H-NS plays in these species. Answering this question requires to determine the structural basis for environmentsensing in H-NS orthologs with drastically different lifestyles. However, the molecular dynamics and multidomain composition of H-NS hamper conventional structural analysis. Therefore, we combined large-scale molecular simulations and spectroscopic approaches to elucidate how environment-sensing by $\mathrm{H}-\mathrm{NS}$ may have adapted in different species. This pluridisciplinary approach yielded an atomic-level understanding of how $\mathrm{H}-\mathrm{NS}$ orthologs evolved specific residue substitutions to adapt environment-sensing to their bacterial habitats, and may open new avenues for strategies to combat antibiotic resistance.

\section{RESULTS}

To investigate the adaptation of environment-sensing by $\mathrm{H}-\mathrm{NS}$, we selected four $\mathrm{H}-\mathrm{NS}$ orthologs from 3000 H-NS-like sequences available in the Uniprot database: 1) H-NSst from $S$. 
typhimurium (a pathogen of mammals), 2) H-NSEA from Erwinia amylovora (a plant pathogen that infects apples and pears), 3) H-NSBA from Buchnera aphidicola (an endosymbiont of aphids), and 4) H-NSIL from Idiomarina loiheinsis (a free-living bacterium from a deep-sea hypothermal vent).

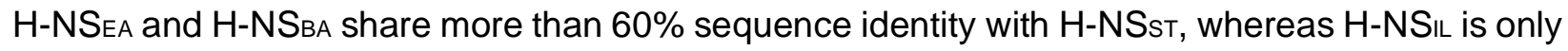
$40 \%$ identical to H-NSst (Fig. 1A). Across the orthologs, the least conserved regions are residues 45-56 in $\alpha 3$, and residues 77-86 (end of $\alpha 4$ and beginning of the linker). The variable residues on $\alpha 3$ and linker may act as simple spacers (Fig. 1), suggesting $\alpha 4$ as a prime candidate for mediating adaptations to the environment.

The site1 dimer is markedly more stable than the site2 dimer in the H-NS orthologs. H-NSsT site1 and site2 form homodimers to enable H-NS multimerization in a head-to-head/tail-to-tail fashion (Fig. 1C) (15). In concert with the DNA interaction of the individual domains, this homooligomerization is required for tight DNA binding and hence gene repression. In our previous study, we showed that only H-NSst site2 dimers unfold and dissociated within a biologically relevant temperature range, whereas site 1 dimers remain unaffected (17). The higher stability of the site1 dimer of H-NSst is explained by a substantially larger contact surface between the two monomers (ca. 3,300 $\AA_{2}$ compared to ca. $850 \AA_{2}$ for site 1 and site2, respectively, according to PDBePISA (27)).

To investigate whether this mechanism is conserved in other H-NS orthologs, we built homology models for $\mathrm{H}-\mathrm{NS} \mathrm{EA}_{\mathrm{A}}, \mathrm{H}-\mathrm{NS} \mathrm{BA}_{\mathrm{B}}$, and H-NSIL using the crystal structure of the H-NSst site1site2 fragment as a template (PDB ID: 3NR7) (15). Next, we constructed a tetrameric model as a minimal representation that conserves all features of the $\mathrm{H}-\mathrm{NS}$ multimer. This tetramer contained two full-length H-NS monomers (residues 1-137, with templates PDB IDs: 3NR7 and 2L93) and two partial monomers, truncated before site2 (residues 1-52) (Fig. 1C). To probe differences in environmental responses of the orthologs, we first used conventional full-atom molecular dynamics (MD). We simulated all four tetramers (a 100,000 atom system; see Methods) for 200 ns at three different conditions $\left(0.15 \mathrm{M} \mathrm{NaCl}, 293 \mathrm{~K} ; 0.50 \mathrm{M} \mathrm{NaCl}, 293 \mathrm{~K} / 20{ }^{\circ} \mathrm{C}\right.$; or $0.15 \mathrm{M} \mathrm{NaCl}$, $313 \mathrm{~K} / 40{ }^{\circ} \mathrm{C}$ ) (Supplementary File 1A).

The tetramer simulations at $0.15 \mathrm{M} \mathrm{NaCl}$ and $293 \mathrm{~K}$ produced a lower residue fluctuation level in site1 (local root-mean-squared fluctuation, RMSF 0.4 to $1.9 \AA$ ) than in site2 (local RMSF 0.5 to $4.4 \AA$ ) for all four orthologs (Supplementary File 1B). The higher stability of the site1 dimer is explained by the generally higher number of nonpolar contacts than in the site2 dimer (Fig. 2, Figure Supplement S1A). These contacts involved conserved hydrophobic amino acid residues, notably L5 (or 15) and L8 of $\alpha 1$, L14 of $\alpha 2$, and L23, L26, V36, and V37 (or I37) of $\alpha 3$ (Fig. 2). 
These interactions remained formed in all site1 dimers in our tetramer simulations $($ at $0.15 \mathrm{M} \mathrm{NaCl}$ at $293 \mathrm{~K}$ ) and tetramer simulations at higher salinity (at $0.50 \mathrm{M} \mathrm{NaCl}$ at $293 \mathrm{~K}$ ) or higher temperature (at $0.15 \mathrm{M} \mathrm{NaCl}$ at $313 \mathrm{~K}$ ). Hence, we found that the stability of the site1 dimers resulted mainly from strong and conserved nonpolar packing. Collectively, these data conclude that the mechanism observed for $\mathrm{H}-\mathrm{NS}$ st — where site1 remains stable, and the site2 stability is affected by the environment - is conserved in H-NSEA, H-NSBA, and H-NSIL.

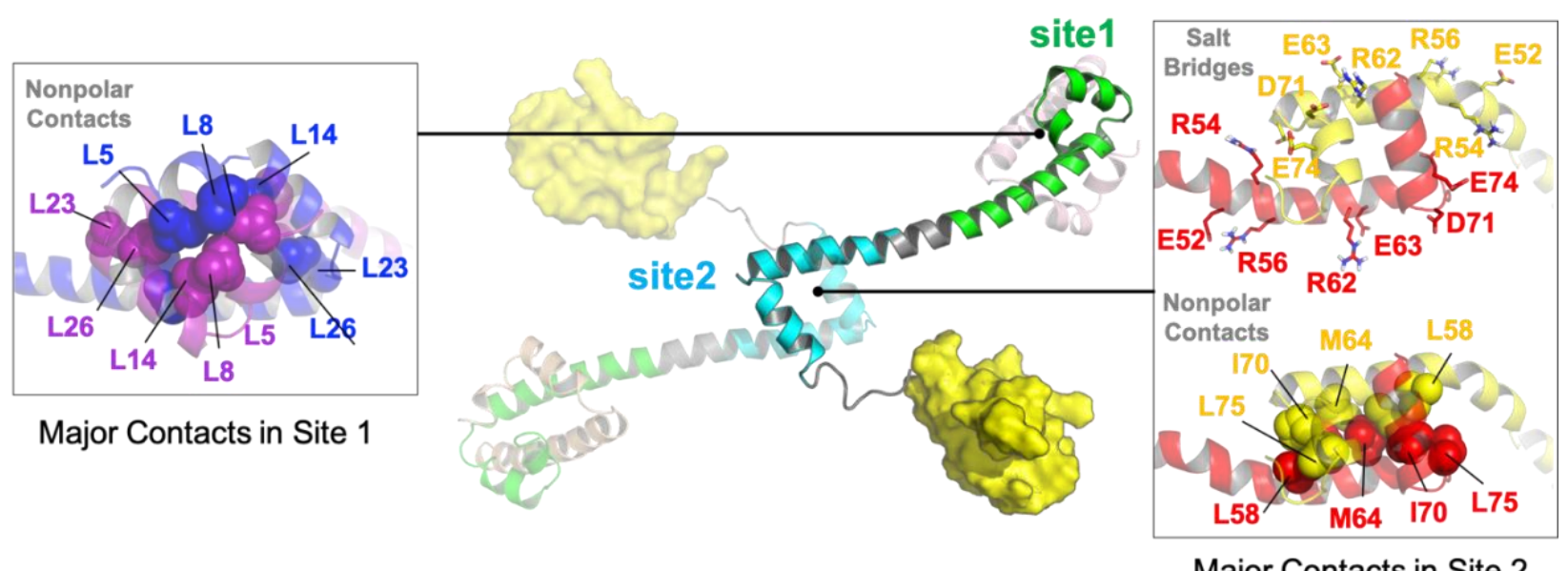

Figure 2. Contacts in site1 and site2 dimers in H-NSst. Hydrophobic contact residues are shown as sphere models, and polar contact residues as stick models. Two protein chains forming the dimer are color-coded. For additional details see Figure Supplement S1.

Variations in the site2 sequence alter the sensing sensitivity of H-NS orthologs. Compared to site1 dimers, site2 dimers harbor fewer nonpolar contacts, only involving residues L58 (or I58), Y61 (or F61), M64 (or A64), I70, and L75 (or 175) (Fig. 2; Figure Supplement S1A). Hence, while site1 dimerization was largely maintained by nonpolar packing, site2 dimerization was strongly driven by electrostatic interactions from selective salt bridges. MD simulations revealed that these salt bridges were in a dynamic equilibrium between forming, breaking, and rearranging. These salt bridges were either formed in cis, within the site2 monomer (e.g., E52-R56 and R62-E63 in H-NSst) or in trans, between two monomers in the site2 dimer (e.g., R54-D71', R54-E74' and K57-D68' in H-NSst; where the apostrophe denotes residues from the second chain; illustrated in Fig. 3). In addition to substitutions that delete (E52A in H-NSBA; E63S and D68A in H-NSIL) or weaken (E63Q in H-NSEA; D71N in H-NSBA) these salt bridges, our simulations showed different levels of site 2 salt bridge stability among orthologs (Fig. 3, Supplementary File 1C): (i) The intermonomer salt bridge R/K54-E/D74' was stable in all our simulations at $293 \mathrm{~K}$ and $0.15 \mathrm{M} \mathrm{NaCl}$, 
but less likely to form at an increased temperature $\left(313 \mathrm{~K} / 40{ }^{\circ} \mathrm{C}\right)$ or salinity $(0.50 \mathrm{M} \mathrm{NaCl})$, suggesting that this salt bridge is involved in environmental sensing (Fig. 3A). (ii) Absent in $\mathrm{H}$ NSIL, the inter-monomer salt bridge K57-D68' remained formed during all our simulations of $\mathrm{H}$ NSst, H-NSEA, and H-NSBA, indicating a 'housekeeping' role for the stability of the site2 dimer in all orthologs except for H-NSIL (Fig. 3B).

Our simulations show how specific protein dynamics might modulate the ortholog's response to salinity or temperature. For example, we observed increased bending of the $\alpha 3$ backbone (annotated by the black arrow in Fig. 3C) at high temperature (313 K) or high salinity $(0.50 \mathrm{M} \mathrm{NaCl})$ (Figure Supplement S2). Although $\alpha 3$ bending occurred in all orthologs, it only significantly affected the site2 dimer of H-NSst by separating R54 from E74' or D71', suggesting that this mechanism contributed to the salt and temperature sensitivity of H-NSst site2, whereas it was not strong enough to significantly affect site2 stability in other orthologs.

Another example was given by H-NSEA, where an alternative R54-D71' salt bridge formed whenever the R54-E74' contact was broken at $313 \mathrm{~K}$. This alternative R54-D71' salt bridge stabilized the H-NSEA site2 dimer at the higher temperature, suggesting that this compensatory mechanism resulted in a decreased sensitivity to temperature (Fig. 3C). H-NSIL provided a final example for a specific response. Compared with the R54-E74' salt bridge (Fig. 3A), the K57-D68' salt bridge only varied slightly in all our simulations (Fig. 3B). However, the substitution D68A in $\mathrm{H}-\mathrm{NS}$ IL supplanted the electrostatic interaction with a nonpolar interaction, which was broken at $313 \mathrm{~K}$ in our simulations (Fig. 3D). This effect suggested that $\mathrm{H}-\mathrm{NS}$ IL had a reduced sensitivity to salinity, while remaining sensitive to temperature. 

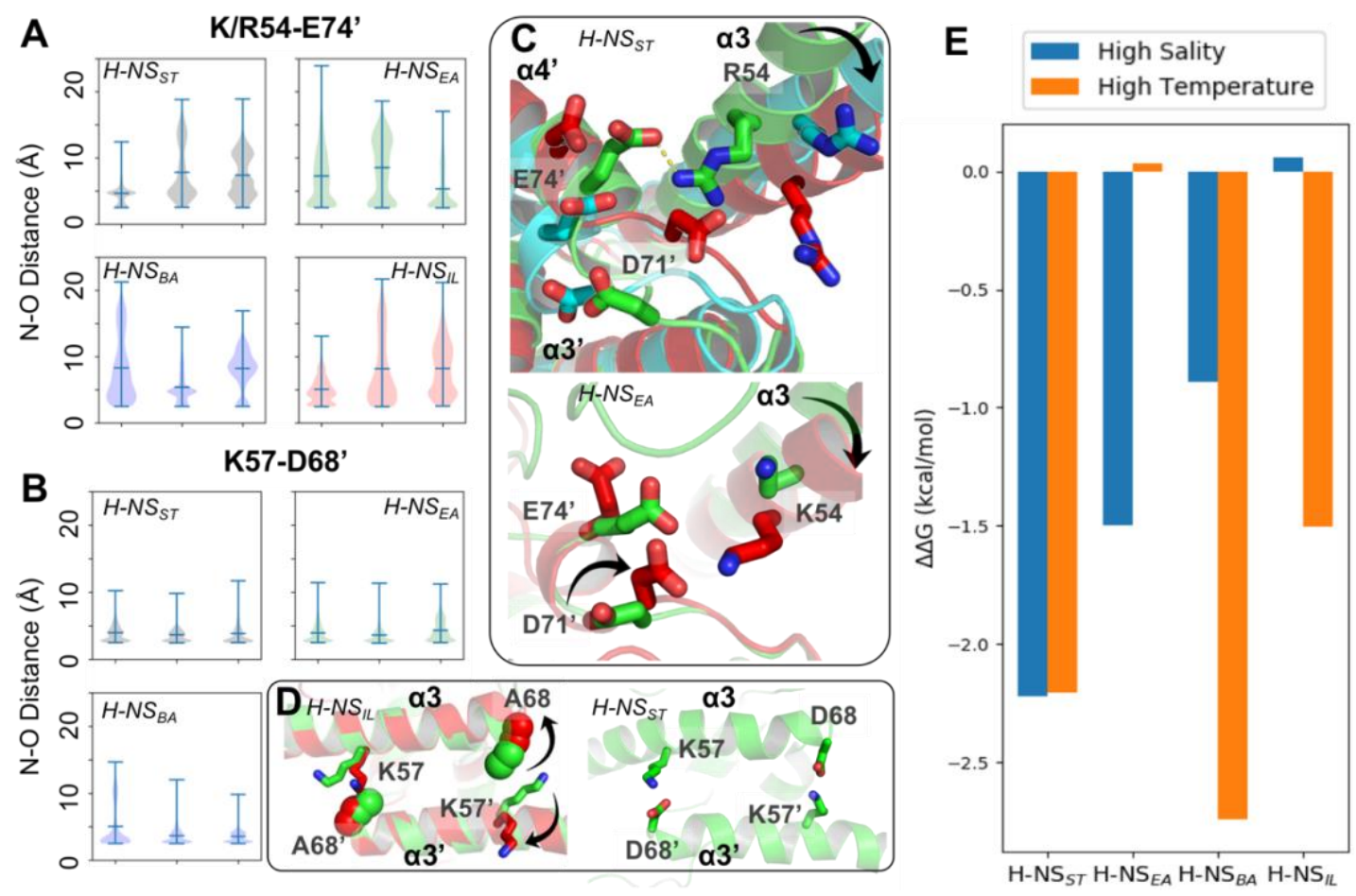

Figure 3. Conserved inter-dimer salt bridges observed in MD simulations. (A-B) Violin plots of the distance between the nearest sidechain nitrogen atom of lysine/arginine and the sidechain oxygen atom of aspartic/glutamic acid in the salt bridge. Each subplot shows the results obtained at $293 \mathrm{~K}, 0.15 \mathrm{M} \mathrm{NaCl}$ (left), $313 \mathrm{~K}, 0.15 \mathrm{M} \mathrm{NaCl}$ (middle), and $293 \mathrm{~K}, 0.50 \mathrm{M} \mathrm{NaCl}$ (right). Each "violin" displays the mean value (the bar in the center of the violin), the range (the stretched line), and the distribution of the distance (kernel density on the side). As we use the numbering of $\mathrm{H}-\mathrm{NSST}$, there are position shifts in H-NSBA and H-NSIL: R54 to R53, K57 to K56 and D68 to D67 in H-NSBA; R54 to K53 in H-NSIL. (C) Final snapshots of the R54E74' salt bridge in H-NSst and K54-D71' in H-NSEA (s. Figure Supplement $\mathbf{S 2}$ for additional details). Color scheme of the cartoon: $293 \mathrm{~K}, 0.15 \mathrm{M} \mathrm{NaCl}$ (green), $313 \mathrm{~K}, 0.15 \mathrm{M} \mathrm{NaCl}$ (red), and $293 \mathrm{~K}, 0.50 \mathrm{M} \mathrm{NaCl}$ (cyan). (D) Final snapshots of the K57-D/A68 contact in H-NSst and H-NSIL. Same color scheme as (C). (E) Free energy changes as a result of increased salinity or temperature, according to the PMFs calculated from umbrella sampling. (s. Figure Supplement S3 for additional details).

To complement the dynamics of H-NS orthologs from our conventional MD simulations, we used extensive simulations with umbrella sampling to quantitate the overall site2 stability. We calculated the potential of mean force (PMF) for site2 dimer dissociation (residues 50-82, ca. 46,000 atoms) of the four H-NS orthologs for three different conditions (low salinity/low temperature, high salinity, or high temperature). The site 2 monomers were not constrained and remained structurally flexible during the dissociation process. To ensure convergence in the PMFs, we employed long windows (54 ns) in simulations totalling $52 \mu$ s (details provided in the 
SI; see Figure Supplement S3 for resulting histograms and PMFs along the dissociation coordinate). According to the free energy difference between the dimerization and dissociation states $\left(\Delta G=G_{\text {dimer- }} G_{\text {dissociation}}\right)$, we estimated the energetic impact from increased salinity and

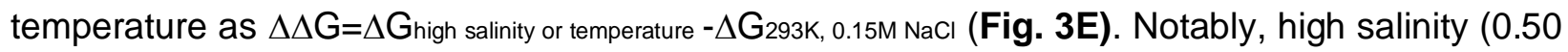
$\mathrm{M} \mathrm{NaCl}$ ) or temperature $(313 \mathrm{~K}$ ) decreased the stability of the H-NSst site2 dimer by $2.2 \mathrm{kcal} / \mathrm{mol}$. $\mathrm{H}-\mathrm{NS}_{\mathrm{BA}}$ displayed a similar sensitivity to temperature but a lower sensitivity to salinity, which destabilized the dimer by $1.5 \mathrm{kcal} / \mathrm{mol}$. Interestingly, our data indicated that H-NSEA was only sensitive to salinity, whereas raising the temperature had little impact on the stability of the $\mathrm{H}$ NSEA site2 dimer. Conversely, H-NSIL only responded to temperature, whereas the increased salinity did not affect the stability of its site2 dimer $(\Delta \Delta \mathrm{G} \sim 0 \mathrm{kcal} / \mathrm{mol})$. Collectively, our conventional MD simulations and PMF calculations suggested how, on the atomic level, changes in the site2 sequence may alter the sensitivity of the H-NS orthologs to different environmental changes.

The autoinhibited $\mathrm{H}-\mathrm{NS}$ conformation is maintained through dynamic electrostatic interactions. In a previous study (17), we had shown that melting and dissociation of site2 dimers allow H-NSst to adapt a closed conformation in which the linker-DNAbd fragment interacts with a negatively charged region on site $1 \alpha 3$ (Figure Supplement S4A), and that this auto-inhibitory interaction is incompatible with DNA interactions. However, due to extensive signal broadening of mainly linker amides exchanging with water, our conventional proton-detected NMR analysis based on exchangeable amide $\mathrm{H} / \mathrm{N}$-observed correlations did not allow confident mapping of the binding site on the C-terminal region (17)( Figure Supplement S4B). Herein, we overcame this limitation by using proton-less ${ }_{13} \mathrm{C}$-detected NMR analysis to complete the resonance assignment of the linker-DNAbd fragment (Fig. 4 and Figure Supplement S4C). These complete carbon chemical shifts allowed us to elucidate the structural mechanism of H-NSst autoinhibition fully, and, in a second step, to use this understanding to investigate the existence of this closed conformation in the orthologs.

We first titrated unlabelled $\mathrm{H}-\mathrm{NS} s \mathrm{~s}$ site1 (residues 1-57) onto the ${ }_{13} \mathrm{C},{ }_{15} \mathrm{~N}$-labelled $\mathrm{H}-\mathrm{NS} s \mathrm{~T}$ C-terminal region (Ctst, residues 84-137), comprising the linker (residues 84-93) and DNAbd (residues 94-137) (Fig. 4A and Figure Supplement S4A). Motif identification from chemical shifts (28) revealed that the interaction promoted the formation of a short type VIII $\beta$-turn in residues 89-92 (Fig. 4B-C). This sharp turn brings the positively charged linker sidechains K87, $\mathrm{K} 89, \mathrm{R} 90, \mathrm{R} 93$ closer to each other than in the free state, presumably as a result of pairing them with opposite charges on site1 (17) (Fig. 4B-C). 
To further probe the local dynamics of the polypeptide chain, we determined the random coil index order parameter $\mathrm{RCI}-S_{2}$ based on the fully assigned ${ }_{13} \mathrm{C}$-resonances for each residue for the ligand-free and site1-saturated Ctst. The dynamics of the well-ordered DNAbd domain remained unchanged with or without site1 present, in agreement with its only minor involvement in the auto-association (Fig. 4D). Conversely, the linker residues 84-95 were disordered without regular secondary motifs in the absence of site $1\left(\mathrm{RCl}-S_{2}<0.35\right)$. Upon addition of site1, the local dynamics decreased, particularly within the stretch of four amino acids K98-R90-A91-A92 (RCI$S_{2}>0.6$ ) that form the type VIII $\beta$-turn according to MICS. Nonetheless, the overall RCI- $S_{2}$ of the linker remained low, demonstrating that the association with site1 did not substantially restrict the linker's movements.

Collectively, our analysis established that the autoinhibitory site1:Ctst association was driven by oppositely charged residues located on site1 and the linker, and involved only a small region of the DNAbd. The resulting intramolecular interaction was maintained through 'fuzzy' charge-pairing that did not fix the partners into a structurally stable complex.
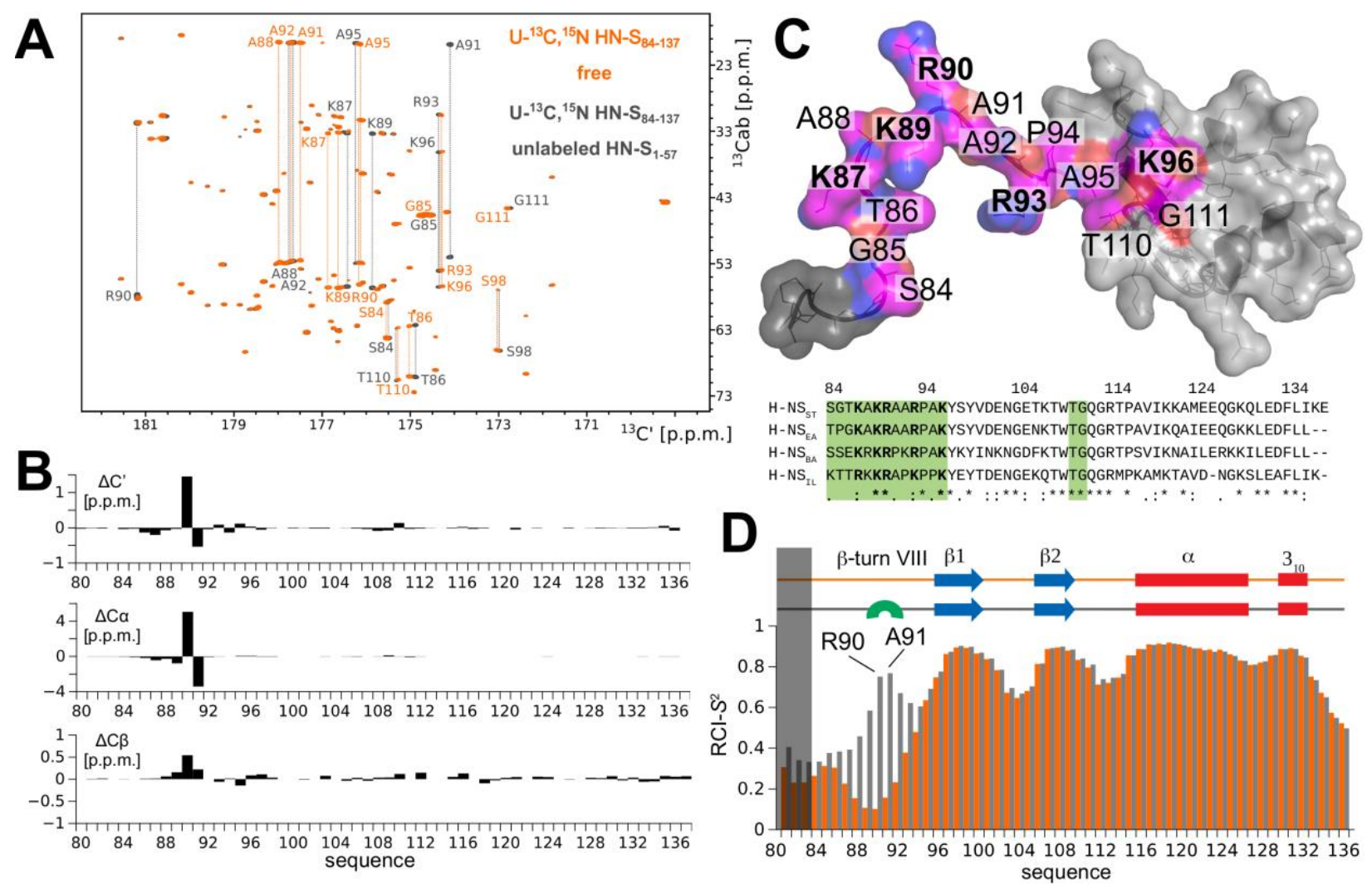

Figure 4. The atomistic details of $\mathrm{H}-\mathrm{NS}$ auto-inhibition revealed by high-resolution proton-less low- $\mathrm{Y}$ detected NMR. (A) The 2D CBCACO correlation ${ }_{13} \mathrm{C}$-detected spectra of ${ }_{13} \mathrm{C},{ }_{15} \mathrm{~N}$ H-NSsTCt (orange) and ${ }_{13} \mathrm{C},{ }_{15} \mathrm{~N}$ H-NSstCt saturated 1:10 (molar) with unlabelled H-NS ${ }_{1-57}$ (dark grey). The OX axis holds all of the ${ }_{13} \mathrm{C},{ }_{15} \mathrm{~N}$ H-NSstCt backbone C' carbonyl chemical shifts correlated with OY (marked ${ }_{13} \mathrm{Cab}$ ) where each 
amino acid stripe crosses with its own $\mathrm{C} \alpha$ and $\mathrm{C} \beta$. NMR chemical shift assignments: BMRB IDs 50239 (apo) and $50240 \mathrm{H}-\mathrm{NS}$ st site1-bound. (B) Top panel: The ${ }_{13} \mathrm{C}$ chemical shift differences as a function of residue number of $\mathrm{H}-\mathrm{NS}_{1-57}$ saturated ${ }_{13} \mathrm{C}_{15} \mathrm{~N} \mathrm{H}-\mathrm{NS} s \mathrm{Ct}$ and apo form. The most marked changes occur in residues K89, R90, A91, and $A 92$ that form a $\beta$-turn type VIII. All residues experiencing significant ${ }_{13} \mathrm{C}$ chemical shift changes upon binding to $\mathrm{H}-\mathrm{NS}_{1-57}$ are marked in magenta on the structure of the $\mathrm{H}-\mathrm{NSsTCt}$ (based on PDBID: 2L93, but extended to contain the full sequence of our construct); positive residues ( $R+K$ ) are labelled in bold. Bottom panel: The sequence alignment of the four selected HN-S orthologs highlighting conserved positively charged linker residues (bold) and residues implicated in binding to site1 (green). (D) Secondary structure motifs (red: helix; blue: $\beta$-sheet; green: $\beta$-turn, present only in the complex) and the $\mathrm{RCl}-\mathrm{S}_{2}$ order parameter (describing the backbone dynamics) of ligand-free (orange) and saturated (gray) H-NSstCt are shown. For additional details, see Figure Supplement S4.

Autoinhibition varies among $\mathbf{H}-\mathrm{NS}$ orthologs. Having established the detailed autoinhibitory interactions between site 1 and the $\mathrm{Ct}$ region in $\mathrm{H}-\mathrm{NS}$ st, we next examined the $\mathrm{H}$ NS orthologs. Based on our structural models (initial homology models and models from conventional MD), the electrostatic surface of the $\mathrm{Ct}$ was well conserved across all H-NS orthologs (Fig. 5A). This level of conservation was expected, given that this region is also required for DNA association (16) - a role that needs to be conserved in all H-NS. Conversely, the site1 surface that binds to $\mathrm{Ct}$ was not conserved across all orthologs. While $\mathrm{H}-\mathrm{NS}$ EA was similar to $\mathrm{H}$ NSst in the overall charge distribution, $\alpha 3$ of H-NSBA showed a distinctly basic surface. H-NSIL displayed an intermediate electrostatic character, with features closer to H-NSst/H-NSEA (Fig. 5A). These findings indicated that the stability of the closed conformation varies across orthologs. To test this prediction, we carried out in vitro binding experiments using microscale thermophoresis (MST).

In vitro binding experiments between site1 and $\mathrm{Ct}$ corroborated that the strength of the auto-association was similar for H-NSst and H-NSEA (Fig. 5B). H-NSBA has mostly lost its capacity for auto-association, in agreement with its altered site1 surface characteristic. Moreover, $\mathrm{H}$ NS ${ }_{B A C t}$ has a proline residue (P91) in position 3 of the $\beta$-turn region, which is highly unfavorable for this secondary structure (29). Conversely, the auto-association was tenfold stronger in $\mathrm{H}-\mathrm{NSIL}$ than in H-NSst, despite a less acidic site1 and despite the presence of a mildly unfavourable proline in $\beta$-turn position 4 (P92). Hence, autoinhibition in H-NSIL might include additional and/or different interactions. Increasing the temperature decreased the self-association strength 2-3 fold in $\mathrm{H}-\mathrm{NS} s \mathrm{a}$ and $\mathrm{H}-\mathrm{NS} \mathrm{LL}$, and more than tenfold in $\mathrm{H}-\mathrm{NS}$ EA (Fig. 5B). It also decreased the $\mathrm{Kd}_{d}$ for $\mathrm{H}$ NS ${ }_{B A}$ to values beyond the measurement range. We concluded that the strength of the autoinhibitory conformation is mostly modulated by the electrostatic surface characteristics of 
site1, with additional influence from $\beta$-turn-breaking proline residues in the $\mathrm{Ct}$, which otherwise preserves its basic characteristic needed for DNA interactions.
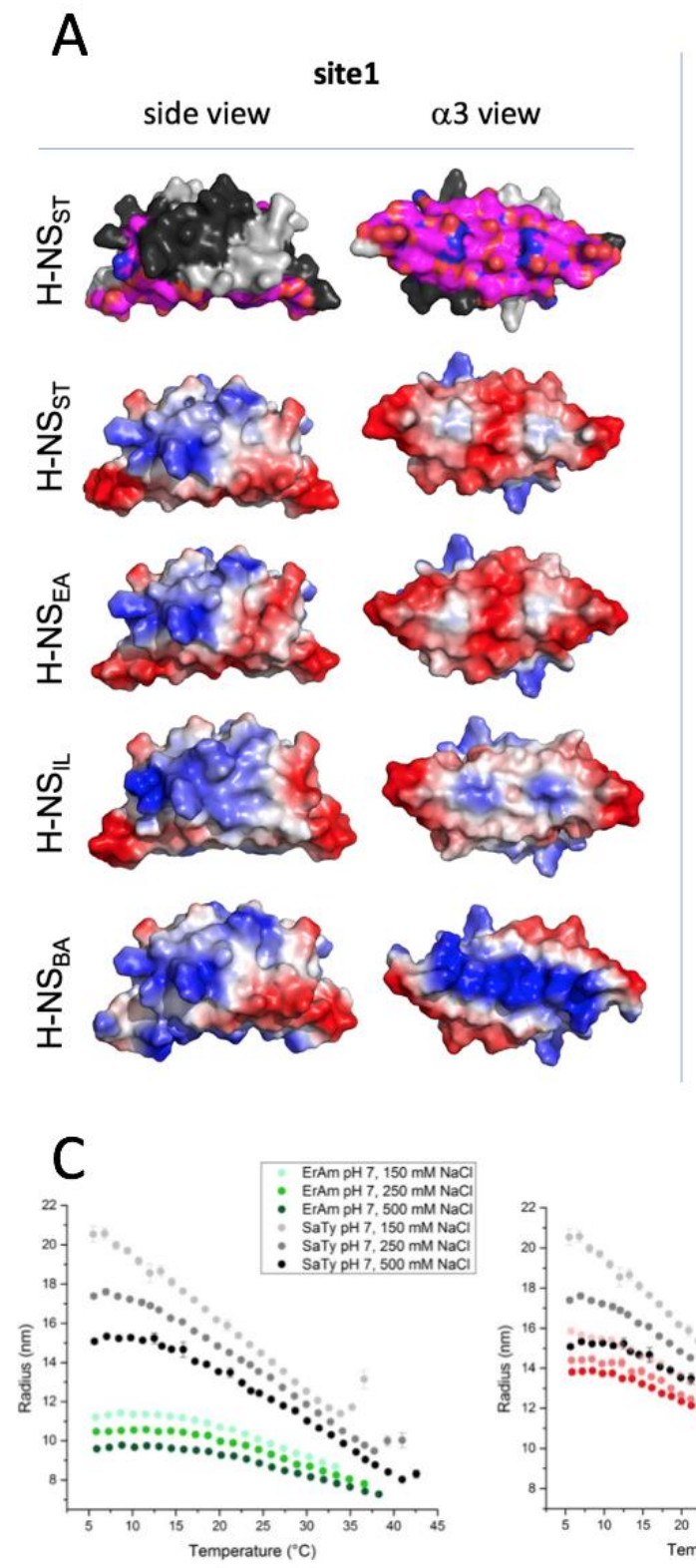

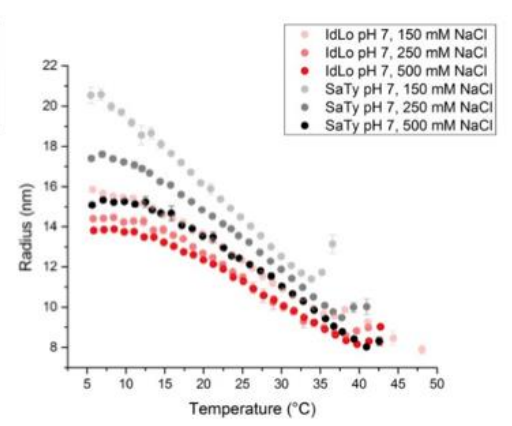

$\mathrm{B}$
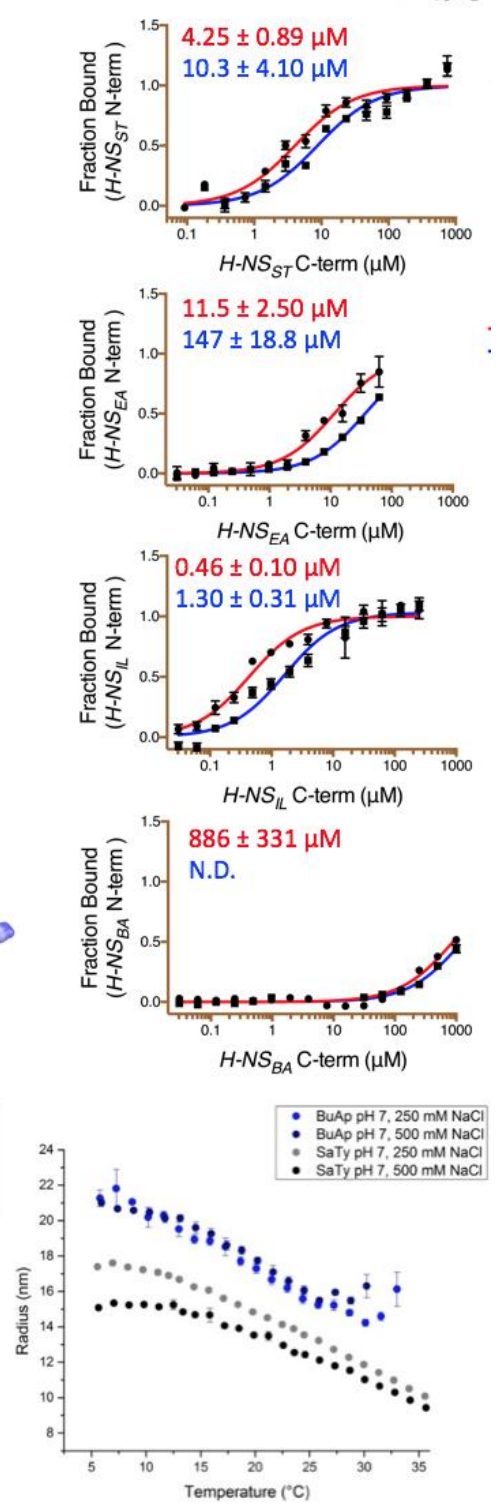

Figure 5. Modeling and in vitro analysis of autoinhibition and multimerization of H-NS orthologs. (A) Left and middle panel: Surface representation of site1 of $\mathrm{H}-\mathrm{NS}$ orthologs (modeled on E. coli H-NS, PDB accession 1NI8) shown as side and $\alpha 3$ (bottom) view. Right panel: Ct, comprising the linker-DNAbd fragment, residues 83-137. The top row shows the residues mapped by NMR involved in forming the autoinhibitory closed conformation ((17) and this study). Other rows show the electrostatic surfaces, colorramped from blue (positive) to red (negative) (calculated and visualized by Pymol). (B) Microscale Thermophoresis (MST) titrations of unlabelled Ct onto $50 \mathrm{nM}$ of alexa488-labelled site 1 at $23{ }^{\circ} \mathrm{C}$ (red) and 
$40{ }^{\circ} \mathrm{C}$ (blue). The dissociation constant $K_{d}$ is color-coded in red $\left(23^{\circ} \mathrm{C}\right)$ and blue $\left(40^{\circ} \mathrm{C}\right)$. N.D.: not determined. (C) DLS experiments showing changes in hydrodynamic radius (as a proxy of apparent site2 stability) upon changes in salinity and temperature. Data in (B,D) are means \pm S.D., $n=3$.

H-NS orthologs show adaptative features in vitro. We next experimentally assessed the response of the $\mathrm{H}-\mathrm{NS}$ orthologs to physicochemical changes using dynamic light scattering (DLS). DLS provides the average hydration radius $R_{H}$ of the particles in solution, and hence gives a proxy for the tendency of H-NS molecules to form site2-mediated multimers or (still site1-linked) dimers. Thus, the $R_{H}$ is a convoluted signal of both effects, i.e., the relative strength of site1 multimerization and of the auto-inhibitory conformation (if it exists). We measured the $R_{H}$ under different salt concentrations and temperatures. As reported previously, H-NSst showed a clear drop in $R_{H}$ from 10 to $40{ }^{\circ} \mathrm{C}$ (Fig. 5C) (17). The marked decrease of $R_{H}$ for curves at 0.15, 0.25, and $0.50 \mathrm{M} \mathrm{NaCl}$ indicated a strong inverse correlation between salinity and site 2 stability.

All three H-NS orthologs displayed a similar behaviour overall, further supporting that the general mechanism of site2-mediated multimerization and environment sensing was preserved. However, we noted important differences in the orthologs' response characteristics (Fig. 5C): (i) Of the four orthologs, H-NSst responded most strongly to salinity and temperature, consistent with the broken R54-E74' salt bridge and large site2 RMSF in our high-salinity or high-temperature simulations. (ii) H-NSEA was less temperature-sensitive and showed weaker multimerization than the other orthologs. Indeed, our simulations suggested that H-NSEA site2 can rearrange the interdimer salt bridge and form either R54-E74' or R54-D71' to maintain site2 stability at higher temperatures. (iii) H-NSBA had the highest tendency to multimerize among all the orthologs tested, which might partly be explained by an absence of the autoinhibitory conformation. Compared to H-NSst, our PMF calculations showed a slightly higher sensitivity to temperature and a slightly reduced sensitivity to salinity. Although these tendencies were apparent in our DLS data, these data were also affected by the fact that $\mathrm{H}-\mathrm{NS}_{\mathrm{BA}}$ required more than $150 \mathrm{mM} \mathrm{NaCl}$ to stay in solution, but $\mathrm{H}-\mathrm{NS}$ BA nonetheless aggregated at $30{ }^{\circ} \mathrm{C}$. (iv) $\mathrm{H}-\mathrm{NS}$ IL showed a decreased sensitivity to salinity compared to H-NSst, as suggested by our computational analysis (i.e., the lack of the site2 K57-R68' salt bridge, the lack of salt-promoted free energy changes, and the attenuated electrostatic site1 surface). Collectively, our experimental observations revealed significant differences in response to physicochemical parameters, which were in agreement with our predictions based on the molecular features of the H-NS orthologs. 
Conclusions. Environment-sensing through the pleiotropic gene regulator H-NS helps $S$. typhimurium to adapt when it is present inside its host mammal. In a previous study, we had shown that an increase in temperature, and to some extent salinity, dissociates the second dimerization element (site2), which produces two effects: firstly, it impedes synergistic DNA binding of $\mathrm{H}-\mathrm{NS}$ multimers, and secondly, it allows $\mathrm{H}-\mathrm{NS}$ to adopt an autoinhibitory conformation where DNA binding residues on the C-terminal linker-DNAbd fragment associate with the $\mathrm{N}$ terminal site1 dimerization domain (17). In this study, we confirmed key aspects of this model, namely that site 2 is the element that senses changes in physicochemical parameters. We also uncovered additional aspects of this process. In particular, proton-less NMR fully revealed the position and dynamics of the linker-DNAbd residues involved in the autoinhibitory association with site1. The $\beta$-turn linker residues 89-91 critical for autoinhibition cannot reach site1 without site 2 dissociation (Figure Supplement S1B), confirming that the closed autoinhibited conformation is mutually exclusive with H-NS multimerization along DNA. Our NMR analysis also demonstrated that this autoinhibition is achieved at a low entropic cost, maintaining a high flexibility with respect to the exact distribution of the interacting charges on both site1 and the linker-DNAbd fragment. On the one hand, avoiding the entropic penalty helps the autoinhibitory interaction to prevail against the competing DNA association. (Of note, the covalent link between site1 and the Ct will enhance their local concentration and hence their apparent affinity compared to our measurement based on separate domains in Fig. 5B). On the other hand, the fuzziness of the charge-charge interactions facilitates preserving the autoinhibition during bacterial evolution and adaptation.

Based on our refined understanding of the molecular details on the stability and dynamics of site 2 and the site 1 :Ct association of $S$. typhimurium H-NS, we then investigated environmentsensing of $\mathrm{H}-\mathrm{NS}$ orthologs from bacteria that infect plants, bacteria that are endosymbionts of insects, and bacteria that are presumably free-living in or close to a hydrothermal vent. Across all four orthologs, we observed a conceptually similar response to temperature and salt, both overall and on an atomic level, where salt bridges play key roles. This similarity suggests that environment-sensing in H-NS evolved by adaptation of an ancestral feature, namely the relative instability of the simple site 2 helix-turn-helix dimerization motif. However, marked idiosyncrasies in the response of $\mathrm{H}-\mathrm{NS}$ orthologs suggest that this ancestral feature was adapted to fit the current habitat and lifestyle. Our combined copmutational and experimental structural analysis allowed us to relate the observed in vitro features to events on a residual level: in particular, the salt bridge disposition and stability of site2, and the strength of the autoinhibition governed mostly by the electrostatics of site1 helix $\alpha 3$. 
Although other factors inside bacteria can modify the in vitro behaviour of the isolated protein, it is interesting to consider these idiosyncrasies with respect to the bacteria's habitats (Fig. 6):

(i) H-NSst had the highest sensitivity to temperature and salt, in agreement with the critical role of H-NSst in helping Salmonella adapt its gene expression profile depending on if it is inside or outside a warm-blooded mammal.

(ii) In comparison, we found that the response to temperature was markedly attenuated in $\mathrm{H}-\mathrm{NS}$ EA. E. amylovora is the causing agent of fire blight, a contagious disease that mostly affects apples and pears (30). The reduced sensitivity of H-NS to temperature may reflect the minor importance of this factor in an environment of ambient temperature in temperate climate zones.

(iii) $B$. aphidicola is an intracellular symbiont of aphids that is maternally transmitted to the next generation via the ovaries (31). B. aphidicola co-evolved with aphids for more than 150 million years, and despite having the highest sequence identity $(61 \%)$ to $\mathrm{H}-\mathrm{NS} s \mathrm{~s}$ of all orthologs, $\mathrm{H}$-NSBA showed the least conserved features among the orthologs tested, indicating that adaptive evolution was achieved by only minor changes. H-NSBA site2 interactions were stronger than those of other orthologs, and the features promoting the autoinhibitory form were compromised. Hence, H-NSBA may provide a stronger and more robust repression of the genes that it controls. In vitro, $\mathrm{H}-\mathrm{NS} \mathrm{B}_{\mathrm{BA}}$ was the least stable ortholog tested, and had already started to aggregate above $30{ }^{\circ} \mathrm{C}$, in agreement with the fact that $B$. aphidicola cannot survive temperatures of $35^{\circ} \mathrm{C}$ for extended periods.

(iv) Despite having a sequence identity least similar to H-NSst (41\%), H-NSIL maintained an overall similar response profile. However, with an aggregation temperature of $45-50{ }^{\circ} \mathrm{C}, \mathrm{H}-\mathrm{NS} \mathrm{IL}$ was the most heat-stable, especially at low $\mathrm{pH}$ and high salinity, as expected for a thermophilic and halophilic bacterium. Moreover, autoinhibition was tenfold stronger than in Salmonella H-NS and relatively little affected by heat. The natural environment of $I$. loiheinsis (hot hydrothermal fluids venting into cold seawater) provides a temperature range from 4 to $163^{\circ} \mathrm{C}$ (32), suggesting that temperature-sensing by $\mathrm{H}-\mathrm{NS}$ IL is biologically relevant. The attenuated response of $\mathrm{H}-\mathrm{NS} I \mathrm{~L}$ to salinity might reflect the capacity of $I$. loiheinsis to grow in $20 \%(\mathrm{w} / \mathrm{v}) \mathrm{NaCl}$ medium.

Our integrative approach provided atomistic insights on how residue-level substitutions on a protein support adaptation of organisms to different lifestyles. These molecular insights into bacterial adaptative evolution may inspire new strategies to treat related human diseases and to combat antibiotic resistance in the future. 
bioRxiv preprint dol: https://doi.org/10.1101/2020.04.21.053520; this version posted April 24, 2020. The copyright holder for this preprint (which was not certified by peer review) is the author/funder, who has granted bioRxiv a license to display the preprint in perpetuity. It is made available under aCC-BY 4.0 International license.

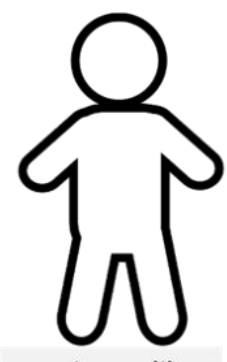

most sensitive to temperature and salinity

HeNS ${ }_{\text {T }}$

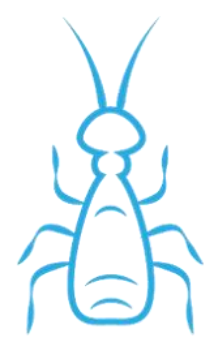

compromised autoinhibition for coevolution

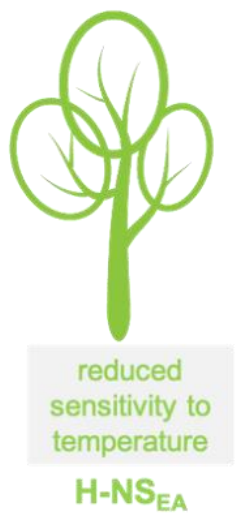

Figure 6. Summary of the most notable adaptations in environment sensing observed for the H-NS orthologs.

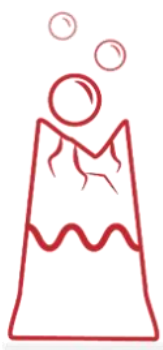

reduced sensitivity to salinity

HeNS ${ }_{\text {IL }}$

16 


\section{METHODS}

\section{Simulation Details.}

Using the CHARMM36 all-atom force field, we performed conventional MD simulations of H-NS tetramers and umbrella sampling of site2 dimers in GROMACS. For DLS and MST, recombinant protein production and measurements were adapted from (17). However, we fluorescently labelled site1 for DLS, instead of the Ct. For NMR, ${ }_{13} \mathrm{C},{ }_{15} \mathrm{~N}$-labelled S. typhimurium H-NS $84-137$ was expressed in minimal M9 media with $5 \mathrm{~g} / \mathrm{L}$ of $\mathrm{U}-{ }_{13} \mathrm{C}$ glucose and $1 \mathrm{~g} / \mathrm{L}$ of ${ }_{15} \mathrm{NH}_{4} \mathrm{Cl}$ salt. Proton and low-y detected high-resolution NMR spectroscopy was carried out on a 700-MH Bruker NEO spectrometer equipped with a 5-mm cryogenic TXO direct observe ${ }_{15} \mathrm{~N},{ }_{13} \mathrm{C}$-optimized probe at 25 ○C. Further details are shown as below.

1.1 Model preparation: We built our homology model of full-length H-NS (UniprotID: P0A1S2) based on orthologues in SwissModel (33) with the templates for the dimerization domain (PDBID: 3NR7) and the DNA-binding domain (PDBID: 2L93). The site2 dimer models were initiated in an anti-parallel configuration, while the tetramer models were constructed according to the crystal packing (PDBID: 3NR7). Maestro (Schrödinger, Inc.) was used to construct the full-length model from different domains.

1.2 Simulation setup: Our simulations were carried out by GROMACS (34) (MD simulations of tetramers and Potential of Mean Force (PMF) simulations of site2 dimers). All the models were solvated in a TIP3P water box, with counterions to neutralize the charges and additional $\mathrm{NaCl}$ for the desired salinity. Each tetramer system contains ca. 33,000 TIP3P water molecules, counter ions, and 150 or $500 \mathrm{mM} \mathrm{NaCl}$, totaling ca. 100,000 atoms in a periodic box $13 \times 9 \times 9 \mathrm{~nm} 3$. All simulations were performed following a minimization, 250 ps equilibration in the NVT and NPT ensemble with Berendsen temperature and pressure coupling, and a production stage NPT (293 or $313 \mathrm{~K}, 1$ bar). The CHARMM36 force field (35) was used with the cmap correction. The particle mesh Ewald (PME) technique (36) was used for the electrostatic calculations. The van der Waals and short-range electrostatics were cutoff at $12.0 \AA$ with switch at $10 \AA$.

The PMF simulations were carried out with the MD program GROMACS (34) using the umbrellaing sampling (US) technique. The CHARMM36 force field was also used. Each site2 monomer of the center of mass (COM) distance was chosen as the disassociation pathway and used for enhanced sampling. After 500 ps equilibrium with the NPT ensemble, initial structures for windows along the reaction coordinates were generated with steered MD. In the steered MD 
simulation, one chain was pulled away along in the direction of increasing the COM distance with force constant of $12 \mathrm{kcal} \mathrm{mol}-1 \AA$-2, until the COM distance reached $25 \AA$. The windows were taken within a range of 0-25 $\AA$. The umbrella windows were optimized at the $0.3 \AA$ interval to ensure sufficient overlap. There are about 80 windows per simulation, and each window was simulated with force constant of $1.2 \mathrm{kcal} \mathrm{mol}{ }_{-1} \AA_{-2}$. All PMF simulations converge in 54 ns per window (Supplementary Fig. S1).

1.3 Computational Data analysis: All the data analyses were carried out in GROMACS, VMD Tcl scripts, and in-house Python programs. In particular, root-mean-square fluctuations (RMSF), root-mean-square deviation (RMSD), salt-bridge interaction, polar interaction, and hydrophobic interaction were analyzed in VMD (37) For salt-bridges, the $\mathrm{O}$ and $\mathrm{N}$ atoms in the charged residues (Arg, His, Lys, Asp, and Glu) were used with a distance cut-off of $4.5 \AA$. For the polar interaction, the atoms in the side chains with the partial charge cutoff (> 0.3 unit for a polar contact) were used with a distance cutoff of $4.5 \AA$. The cut-off for classifying hydrophobic interactions was $6.0 \AA$ between the $\mathrm{C}$ atoms of the hydrophobic residues. The relative percentage $(P)$ of hydrophobic contacts is defined as Eq. 1.

$$
P=\sum_{n=1}^{N} \frac{C_{n}}{N M}
$$

$\mathrm{N}$ is the total number of frames, $C_{n}$ is the number of hydrophobic contacts at frame $n$, and $M$ is the largest value in the series of $C_{n}$. The PMFs were determined using the Weighted Histogram Analysis Method (WHAM) (38) implemented in GROMACS. All the visualization was performed with python, VMD, Pymol (Schrödinger, Inc.), and Maestro (Schrödinger, Inc.).

\section{Experimental Details.}

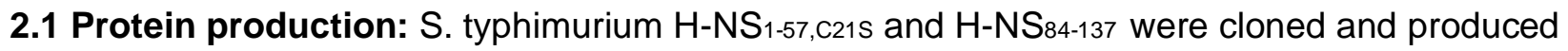
as described previously (17). E. amylovora (H-NS $1-57, \mathrm{H}-\mathrm{NS}_{82-134)}$, B. aphidicola (H-NS $1-57, \mathrm{H}-\mathrm{NS} 84-$ 135) and $I$. loihiensis (H-NS ${ }_{1-57,} \mathrm{H}-\mathrm{NS}_{85-138}$ ) genes were individually cloned into pGEX6P-1, expressed and purified as described previously (17). For the high-resolution nuclear magnetic resonance (NMR) studies the uniformly double ${ }_{13} \mathrm{C},{ }_{15} \mathrm{~N}$-labelled $\mathrm{S}$. typhimurium $\mathrm{H}-\mathrm{NS} 84-137$ (with additional GPLG residues before $\mathrm{S}_{84}$ ) was expressed in minimal M9 media with $5 \mathrm{~g} / \mathrm{L}$ of $\mathrm{U}_{-13} \mathrm{C}$ glucose and $1 \mathrm{~g} / \mathrm{L}$ of ${ }_{15} \mathrm{NH}_{4} \mathrm{Cl}$ salt. The unlabeled $S$. typhimurium $\mathrm{H}-\mathrm{NS}{ }_{1-57} \mathrm{~N}$-terminal domain was expressed and purified as before (17). The final NMR buffer was $50 \mathrm{mM} \mathrm{NaCl}, 2 \%(v / v) \mathrm{D}_{2} \mathrm{O}, 20$ mM Bis-TRIS at $\mathrm{pH} 6.5$ and $0.002 \% \mathrm{NaN}_{3}$. 
2.2 Dynamic Light Scattering: For DLS measurements, H-NS from Salmonella typhimurium, Erwinia amylovora, Buchnera aphidicola and Idiomarina loihiensis were expressed as N-terminal mCherry fusion proteins with an N-term His tag in E. coli BL21 using the expression vector pET28b. The linker sequence SAGGSASGASG was inserted between mCherry and H-NS proteins to avoid steric clashes in the dimer. Bacteria were grown in LB medium, induced with $1 \mathrm{mM}$ IPTG at $25{ }^{\circ} \mathrm{C}$ overnight. Cells were harvested and resuspended in lysis buffer (50 mM Tris pH8, $500 \mathrm{mM} \mathrm{NaCl}, 10 \mathrm{mM}$ Imidazole with addition of lysozyme, DNase I and 1\% triton X-100) and lysed by mild sonication. Proteins and bacterial membranes were separated by centrifugation (30 min, at 15,000 $\times$ g) and the supernatant was applied to Ni-NTA beads (Qiagen) for $2 \mathrm{~h}$. The column was washed thoroughly with $50 \mathrm{mM}$ Tris $\mathrm{pH} 8,500 \mathrm{mM} \mathrm{NaCl}, 10 \mathrm{mM}$ Imidazole and protein was then eluted with $50 \mathrm{mM}$ Tris pH8, $500 \mathrm{mM} \mathrm{NaCl}, 400 \mathrm{mM}$ Imidazole, $1 \mathrm{mM}$ DTT. After dialysis in 50 mM HEPES pH7.4, $300 \mathrm{mM} \mathrm{NaCl}, 0.5 \mathrm{mM}$ TCEP, eluted protein was further purified by ionexchange chromatography using either MonoQ or MonoS column (GE) in the same buffer. Protein multimerization was observed in combination of different salt $(150,250$ and $500 \mathrm{M} \mathrm{NaCl})$ and $\mathrm{pH}$ (6, 7 and 8) conditions. For this, $100 \mathrm{mM}$ MES, MOPS and HEPES buffers were used, with proteins at concentrations ranging from 125 to $500 \mu \mathrm{M}$, in a final volume of $100 \mu \mathrm{L}$. Dynamic light scattering measurements were performed in 96-well plates (Greiner) using a DynaPro plate reader-II (Wyatt Technologies). A triplicate of three wells was measured for every sample with 5 acquisitions of $5 \mathrm{~s}$ for every well. The machine was cooled with gaseous nitrogen, with a starting temperature of $5{ }^{\circ} \mathrm{C}$, followed by an increase to $60{ }^{\circ} \mathrm{C}$ at a ramp rate set so that each well is measured every $1^{\circ} \mathrm{C}$. Data were analyzed with DYNAMICS software (Wyatt Technologies) as Temperature Dependence and exported for further fitting on Origin software using a Logistic Fit. The presented results are mean values with standard error mean determined from the triplicate sample.

2.3 Proton and low-y detected high-resolution NMR spectroscopy: All NMR measurements were done on $700 \mathrm{MH}$ Bruker NEO spectrometer equipped with $5 \mathrm{~mm}$ cryogenic TXO direct observe ${ }_{15} \mathrm{~N},{ }_{13} \mathrm{C}$-optimized probe at $25 \circ \mathrm{C}$. The sequence specific backbone resonance assignments of visible $\mathrm{H} / \mathrm{N}$ correlations on ${ }_{1} \mathrm{H}$-detected spectra of $\mathrm{S}$. typhimurium $\mathrm{H}-\mathrm{NS}{ }_{84-137}$ protein at $200 \mu \mathrm{M}$ concentration in $A p o$ and $\mathrm{H}_{-} \mathrm{NS}_{1-57}$ saturated (1.5 mM) forms were achieved with classical set of triple resonance experiments, i.e. HNCA, HncoCA, HNCO, HNcaCO, HNCACB, CBCAcoNH (39) and previously published assignments (40). The $100 \%$ complete sets of $\mathrm{C} \alpha, \mathrm{C} \beta$ and $\mathrm{C}^{\prime}$ resonances for $A p o$ and $\mathrm{H}-\mathrm{NS}_{1-57}$ saturated (1.5 mM) forms covering the entire protein sequence, together with the residues not visible on $\mathrm{H} / \mathrm{N}$ correlation ${ }_{1} \mathrm{H}$-detected 
experiments (due to amide exchange with water) were achieved with intra-residue 2D $(\mathrm{H}) \mathrm{CACO}$ (c_hcaco_ia3d, 16 scans) and (H)CACBCO (c_hcbcaco_ia3d, 32 scans) supported with sequential (H)CANCO (c_hcanco_ia3d, 96 scans) ${ }_{13} \mathrm{C}$-detected experiments (41). The low- $\mathrm{y}$, so ${ }_{13} \mathrm{C}$-detected experiments mentioned above were started with ${ }_{1} \mathrm{H}$-excitation in order to enhance the sensitivity and recorded in in-phase and anti-phase (IPAP) mode for the virtual decoupling. All spectra were processed in NMRpipe and analyzed in CARA and Sparky software. The random-coil-index order parameters $\mathrm{RCl}-S_{2}$ and secondary motifs, like $\beta$-turn, for $A p o$ and $\mathrm{H}_{-} \mathrm{NS}_{1}$ 57 saturated (1.5 mM) forms were determined from complete lists of $\mathrm{C}_{\alpha}, \mathrm{C}_{\beta}$ (except glycines), $\mathrm{N}$, C' chemical shifts with the TalosN and MICS programs, respectively. NMR chemical shift assignments for the H-NSstCt in its apo and H-NSst site1-bound states are deposited at the BMRB with the IDs 50239 and 50240, respectively.

2.4 Micro Scale Thermophoresis (MST) for protein-protein interactions: H-NS (residue 1-57) from S. typhimurium, E. amylovora, B. aphidicola and I. loihiensis were individually labeled Nterminally with fluorescent Alexa488-TFP (Thermo Scientific) and then unlabeled C-term of those proteins were titrated against Alexa488 labeled $\mathrm{N}$-term correspondingly and the final results were plotted as described previously (17).

\section{ACKNOWLEDGEMENTS}

Research by $\mathrm{UH}, \mathrm{VK}, \mathrm{FH}, \mathrm{AK}, \mathrm{ML}$, LJ, and SA reported in this work was supported by the King Abdullah University of Science and Technology (KAUST) through the baseline fund and the Award No FCC/1/1976-25 from the Office of Sponsored Research (OSR); XZ was partially supported by the ACS Petroleum Research Fund (58219-DNI); CL, JMR, and JL were partially supported by the National Institutes of Health award (R01GM129431). We acknowledge support from the KAUST Bioscience and Imaging core laboratories and the computational resources from the Vermont Advanced Compute Core (VACC) and the Anton supercomputer in Pittsburgh Supercomputing Center (PSC), and thank M. Cusack (KAUST Research Support Services) for editorial help. 


\section{REFERENCES}

1. R. M. Williams, S. Rimsky, Molecular aspects of the E. coli nucleoid protein, H-NS: a central controller of gene regulatory networks. FEMS Microbiol Lett 156, 175-185 (1997).

2. F. C. Fang, S. Rimsky, New insights into transcriptional regulation by H-NS. Curr Opin Microbiol 11, 113-120 (2008).

3. R. S. Winardhi, J. Yan, L. J. Kenney, H-NS Regulates Gene Expression and Compacts the Nucleoid: Insights from Single-Molecule Experiments. Biophysical journal 109, 1321-1329 (2015).

4. S. S. Ali, B. Xia, J. Liu, W. W. Navarre, Silencing of foreign DNA in bacteria. Curr Opin Microbiol 15, 175-181 (2012).

5. B. R. Gordon et al., Structural basis for recognition of AT-rich DNA by unrelated xenogeneic silencing proteins. Proceedings of the National Academy of Sciences of the United States of America 108, 10690-10695 (2011).

6. R. Landick, J. T. Wade, D. C. Grainger, H-NS and RNA polymerase: a love-hate relationship? Curr Opin Microbiol 24, 53-59 (2015).

7. B. Lang et al., High-affinity DNA binding sites for $\mathrm{H}$-NS provide a molecular basis for selective silencing within proteobacterial genomes. Nucleic acids research 35, 6330-6337 (2007).

8. W. W. Navarre, M. McClelland, S. J. Libby, F. C. Fang, Silencing of xenogeneic DNA by HNS-facilitation of lateral gene transfer in bacteria by a defense system that recognizes foreign DNA. Genes Dev 21, 1456-1471 (2007).

9. S. S. Ali et al., Silencing by H-NS potentiated the evolution of Salmonella. PLoS Pathog 10, e1004500 (2014).

10. W. R. Will, W. W. Navarre, F. C. Fang, Integrated circuits: how transcriptional silencing and counter-silencing facilitate bacterial evolution. Curr Opin Microbiol 23, 8-13 (2015).

11. K. Higashi et al., H-NS Facilitates Sequence Diversification of Horizontally Transferred DNAs during Their Integration in Host Chromosomes. PLoS Genet 12, e1005796 (2016).

12. X. Robert, P. Gouet, Deciphering key features in protein structures with the new ENDscript server. Nucleic acids research 42, W320-324 (2014).

13. H. Shindo et al., Solution structure of the DNA binding domain of a nucleoid-associated protein, H-NS, from Escherichia coli. FEBS letters 360, 125-131 (1995).

14. V. Bloch et al., The H-NS dimerization domain defines a new fold contributing to DNA recognition. Nat Struct Biol 10, 212-218 (2003).

15. S. T. Arold, P. G. Leonard, G. N. Parkinson, J. E. Ladbury, H-NS forms a superhelical protein scaffold for DNA condensation. Proceedings of the National Academy of Sciences of the United States of America 107, 15728-15732 (2010).

16. Y. Gao et al., Charged residues in the H-NS linker drive DNA binding and gene silencing in single cells. Proceedings of the National Academy of Sciences of the United States of America 114, 12560-12565 (2017).

17. U. F. Shahul Hameed et al., H-NS uses an autoinhibitory conformational switch for environment-controlled gene silencing. Nucleic acids research 47, 2666-2680 (2019).

18. R. A. van der Valk et al., Mechanism of environmentally driven conformational changes that modulate H-NS DNA-bridging activity. Elife 6, (2017). 
19. W. W. Navarre et al., Selective silencing of foreign DNA with low GC content by the H-NS protein in Salmonella. Science 313, 236-238 (2006).

20. S. S. Ali et al., Structural insights into the regulation of foreign genes in Salmonella by the Hha/H-NS complex. The Journal of biological chemistry 288, 13356-13369 (2013).

21. L. Hu et al., Threonine Phosphorylation Fine-Tunes the Regulatory Activity of Histone-Like Nucleoid Structuring Protein in Salmonella Transcription. Front Microbiol 10, 1515 (2019).

22. T. Oshima, S. Ishikawa, K. Kurokawa, H. Aiba, N. Ogasawara, Escherichia coli histone-like protein $\mathrm{H}$-NS preferentially binds to horizontally acquired DNA in association with RNA polymerase. DNA Res 13, 141-153 (2006).

23. C. A. White-Ziegler, T. R. Davis, Genome-wide identification of H-NS-controlled, temperature-regulated genes in Escherichia coli K-12. J Bacteriol 191, 1106-1110 (2009).

24. C. Kahramanoglou et al., Direct and indirect effects of $\mathrm{H}-\mathrm{NS}$ and Fis on global gene expression control in Escherichia coli. Nucleic acids research 39, 2073-2091 (2011).

25. T. Ueda et al., Functions of the Hha and YdgT proteins in transcriptional silencing by the nucleoid proteins, H-NS and StpA, in Escherichia coli. DNA Res 20, 263-271 (2013).

26. M. V. Kotlajich et al., Bridged filaments of histone-like nucleoid structuring protein pause RNA polymerase and aid termination in bacteria. Elife 4, (2015).

27. E. Krissinel, K. Henrick, Inference of Macromolecular Assemblies from Crystalline State. J Mol Biol 372, 774-797 (2007).

28. Y. Shen, A. Bax, Protein backbone and sidechain torsion angles predicted from NMR chemical shifts using artificial neural networks. Journal of biomolecular NMR 56, 227-241 (2013).

29. T. E. Creighton, Prediction of Protein Structure and the Principles of Protein Conformation. Gerald D. Fasman, Ed. Plenum, New York, 1989. xiv, 798 pp., illus. \$95. Science 247, 1351-1352 (1990).

30. K. Vrancken, M. Holtappels, H. Schoofs, T. Deckers, R. Valcke, Pathogenicity and infection strategies of the fire blight pathogen Erwinia amylovora in Rosaceae: state of the art. Microbiology 159, 823-832 (2013).

31. A. E. Douglas, Nutritional interactions in insect-microbial symbioses: aphids and their symbiotic bacteria Buchnera. Annu Rev Entomol 43, 17-37 (1998).

32. S. P. Donachie, S. Hou, T. S. Gregory, A. Malahoff, M. Alam, Idiomarina loihiensis sp. nov., a halophilic gamma-Proteobacterium from the Lo'ihi submarine volcano, Hawai'i. Int J Syst Evol Microbiol 53, 1873-1879 (2003).

33. K. Arnold, L. Bordoli, J. Kopp, T. Schwede, The SWISS-MODEL workspace: a web-based environment for protein structure homology modelling. Bioinformatics 22, 195-201 (2006).

34. T. A. Wassenaar, H. I. Ingólfsson, M. Prieß, S. J. Marrink, L. V. Schäfer, Mixing MARTINI: electrostatic coupling in hybrid atomistic-coarse-grained biomolecular simulations. The Journal of Physical Chemistry B 117, 3516-3530 (2013).

35. R. B. Best et al., Optimization of the additive CHARMM all-atom protein force field targeting improved sampling of the backbone $\varphi, \psi$ and side-chain $\chi 1$ and $\chi 2$ dihedral angles. Journal of chemical theory and computation 8, 3257-3273 (2012).

36. T. Darden, D. York, L. Pedersen, Particle mesh Ewald: An N $\cdot \log (\mathrm{N})$ method for Ewald sums in large systems. The Journal of chemical physics 98, 10089-10092 (1993). 
37. W. Humphrey, A. Dalke, K. Schulten, VMD: visual molecular dynamics. J Mol Graph 14, 3338, 27-38 (1996).

38. J. S. Hub, B. L. De Groot, D. Van Der Spoel, g_wham-A Free Weighted Histogram Analysis Implementation Including Robust Error and Autocorrelation Estimates. Journal of chemical theory and computation 6, 3713-3720 (2010).

39. M. Sattler, J. Schleucher, C. Griesinger, Heteronuclear multidimensional NMR experiments for the structure determination of proteins in solution. Progress in nuclear magnetic resonance spectroscopy 34, 93-158 (1999).

40. H. Shindo et al., Solution structure of the DNA binding domain of a nucleoid - associated protein, H - NS, from Escherichia coli. FEBS letters 360, 125-131 (1995).

41. F. L. Gray, M. J. Murai, J. Grembecka, T. Cierpicki, Detection of disordered regions in globular proteins using 13C - detected NMR. Protein Science 21, 1954-1960 (2012). 


\section{FIGURE SUPPLEMENTS}

Figure 2 - Figure Supplement S1. The umbrella histogram and convergency to simulate the $\mathrm{H}$ NS orthologs site2 dimer at different simulation conditions: $293 \mathrm{~K}, 0.15 \mathrm{M} \mathrm{NaCl}$ (Green), $293 \mathrm{~K}$, $0.50 \mathrm{M} \mathrm{NaCl}$ (Blue), and $313 \mathrm{~K}, 0.15 \mathrm{M} \mathrm{NaCl}$ (Red). Each PMF subplot contains a series of 5 curves from 38 to 54 ns/window (4 ns increment), demonstrating convergence.

Figure 3 - Figure Supplement S2. Representative H-NS monomers (with site2 alignment) from the final snapshots (at $200 \mathrm{~ns}$ ) of the tetramer simulations under different conditions: $293 \mathrm{~K}, 0.15$ $\mathrm{M} \mathrm{NaCl}$ (green), $293 \mathrm{~K}, 0.50 \mathrm{M} \mathrm{NaCl}$ (cyan), and $313 \mathrm{~K}, 0.15 \mathrm{M} \mathrm{NaCl}$ (red). To highlight the linker region in $\alpha 3$, we use a pale color for the rest of the backbone in each protein. This figure shows that while H-NS proteins are mostly "straight" at $293 \mathrm{~K}, 0.15 \mathrm{M} \mathrm{NaCl}$ (green), bending of a3 is common through all H-NS orthologs under the high-temperature or high-salinity condition. The most significant bending is found in H-NSst.

Figure 3 - Figure Supplement S3. The umbrella histogram and convergency to simulate the $\mathrm{H}$ NS orthologs site2 dimer at different simulation conditions: $293 \mathrm{~K}, 0.15 \mathrm{M} \mathrm{NaCl}$ (Green), $293 \mathrm{~K}$, $0.50 \mathrm{M} \mathrm{NaCl}$ (Blue), and $313 \mathrm{~K}, 0.15 \mathrm{M} \mathrm{NaCl}$ (Red). Each PMF subplot contains a series of 5 curves from 38 to 54 ns/window (4 ns increment), demonstrating convergence.

Figure 4 - Figure Supplement S4. The low- $\gamma{ }_{13} \mathrm{C}$-detected experiments unveil the molecular details of highly dynamic and solvent exposed residues elusive for classical ${ }_{1} \mathrm{H}$-detected approaches. The panel A shows the ${ }_{1} \mathrm{H}-{ }_{15} \mathrm{~N}$ HSQC spectrum of 200 uM ${ }_{13} \mathrm{C},{ }_{15} \mathrm{~N}$-labelled $S$.

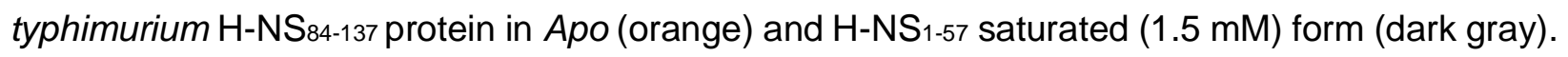
The residues that are missing from $\mathrm{H} / \mathrm{N}$ correlation ${ }_{1} \mathrm{H}$-detected experiments are depicted in panel B with black and green mapped on the NMR solution structure of $\mathrm{H}-\mathrm{NS}$ 91-137 (PDB id 1HNR; the lowest energy structure). The flexible residues of S84-A91 were added together with GPLG artificial residues left after the tag cleavage. The only tryptophan side chain $\mathrm{H} \varepsilon / \mathrm{N} \varepsilon$ imidazole correlation is marked W109 in green. The $\mathbf{C}$ panel presents the overlay of 2D $(\mathrm{H}) \mathrm{CACO}$ and $(\mathrm{H}) \mathrm{CANCO}{ }_{13} \mathrm{C}$-detected experiments and sequential walk for two regions, $\mathrm{G}_{80}-\mathrm{A}_{91}$ marked black and $\mathrm{T}_{110}-\mathrm{G}_{111}$ marked green, that are not detected on $\mathbf{A}$. A complete sequential carbon walk can be done for the entire protein $\mathrm{H}-\mathrm{NS}_{84-137}$ sequence. 


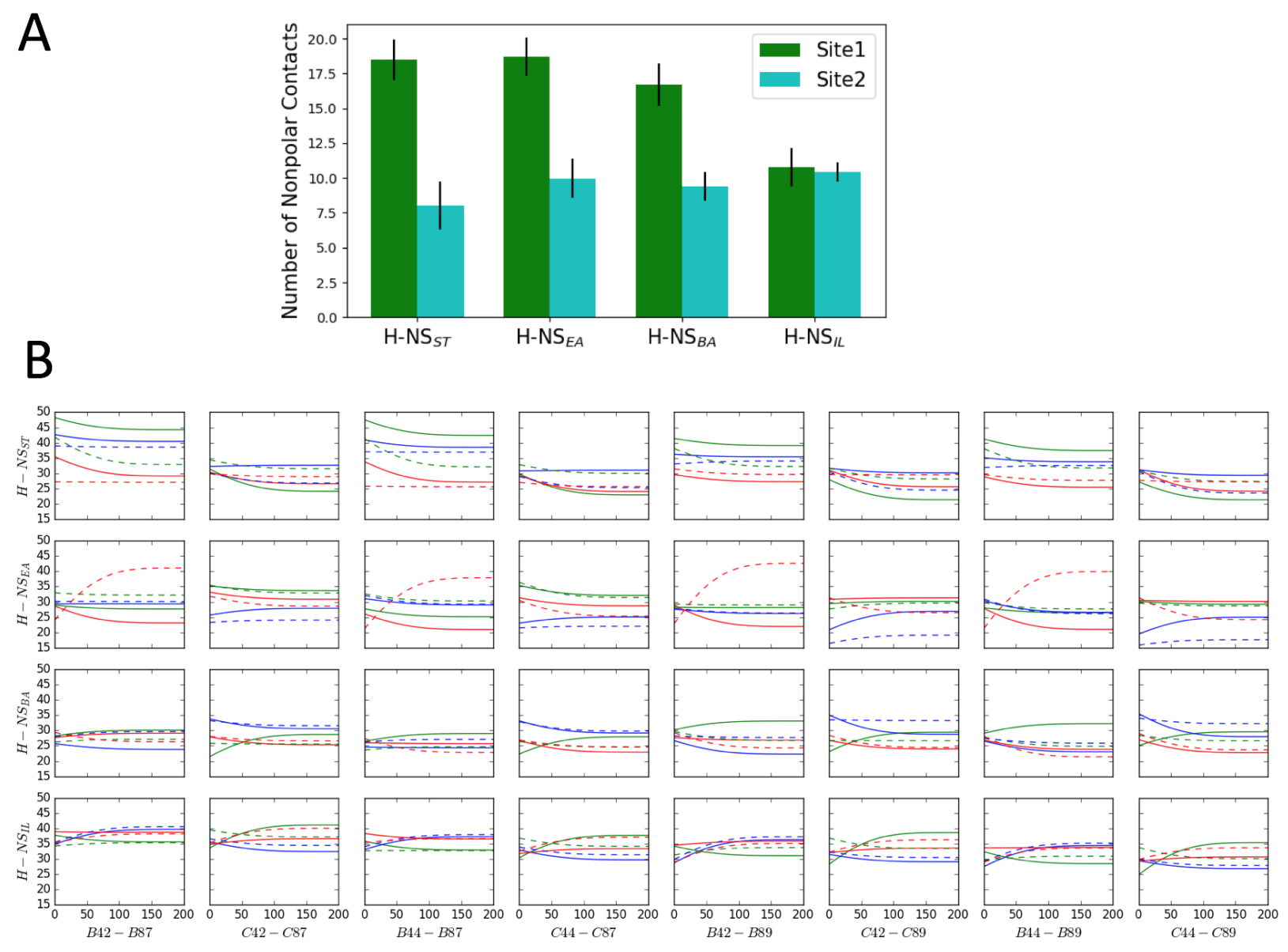

Figure 2 - Figure Supplement S1. (A) Comparison of nonpolar contacts of H-NS orthologues at $293 \mathrm{~K}$ and $0.15 \mathrm{M} \mathrm{NaCl}$, using the last $100 \mathrm{~ns}$ in the $\mathrm{MD}$ simulations. (B) The separation distance between residue $42 / 44$ and residue $87 / 89$ ( $Y$ axis in $\AA$ ) versus time ( $X$ axis in nanosecond). The separation distance was measured as the $C_{\alpha}-C_{\alpha}$ distance in the tetramer model (within chain $\mathrm{B} / \mathrm{C}$, which were modelled as full length). The color scheme annotates different simulation conditions: $293 \mathrm{~K}, 0.15 \mathrm{M} \mathrm{NaCl}$ (Green), $293 \mathrm{~K}, 0.50 \mathrm{M} \mathrm{NaCl}$ (Blue), and $313 \mathrm{~K}, 0.15 \mathrm{M} \mathrm{NaCl}$ (Red). For clarity, we show the smoothed data of two replicas for each system (solid and dash lines respectively). Since complete unfolding of site2 was not observed in these simulations (presumably due to the short timescale and the difficulty of sampling), these plots indicate a minimum separation of 15 to $20 \AA$ between the $\mathrm{N}$ and $\mathrm{C}$ termini, demonstrating that site 2 has to unfold to allow closer contacts between the termini. 

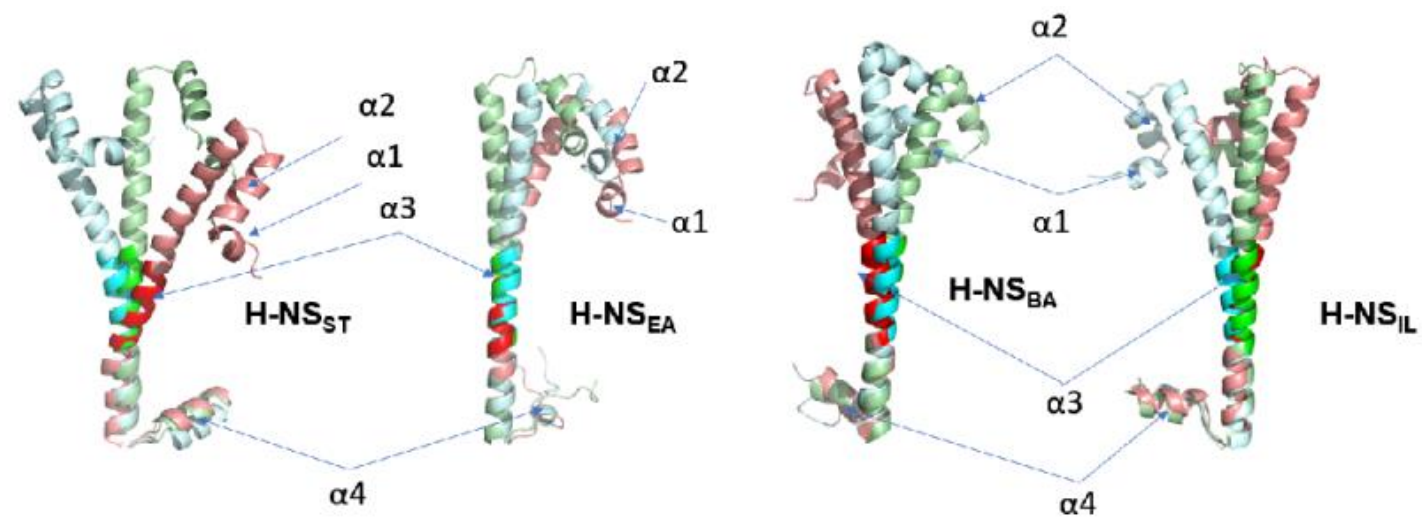

Figure 3 - Figure Supplement S2. Representative H-NS monomers (with site2 alignment) from the final snapshots (at $200 \mathrm{~ns}$ ) of the tetramer simulations under different conditions: $293 \mathrm{~K}$, $0.15 \mathrm{M} \mathrm{NaCl}$ (green), $293 \mathrm{~K}, 0.50 \mathrm{M} \mathrm{NaCl}$ (cyan), and $313 \mathrm{~K}, 0.15 \mathrm{M} \mathrm{NaCl}$ (red). To highlight the linker region in $\alpha 3$, we use a pale color for the rest of the backbone in each protein. This figure shows that while H-NS proteins are mostly "straight" at $293 \mathrm{~K}, 0.15 \mathrm{M} \mathrm{NaCl}$ (green), bending of a3 is common through all H-NS orthologs under the high-temperature or high-salinity condition. The most significant bending is found in $\mathrm{H}-\mathrm{NS} \mathrm{S}_{\mathrm{ST}}$. 

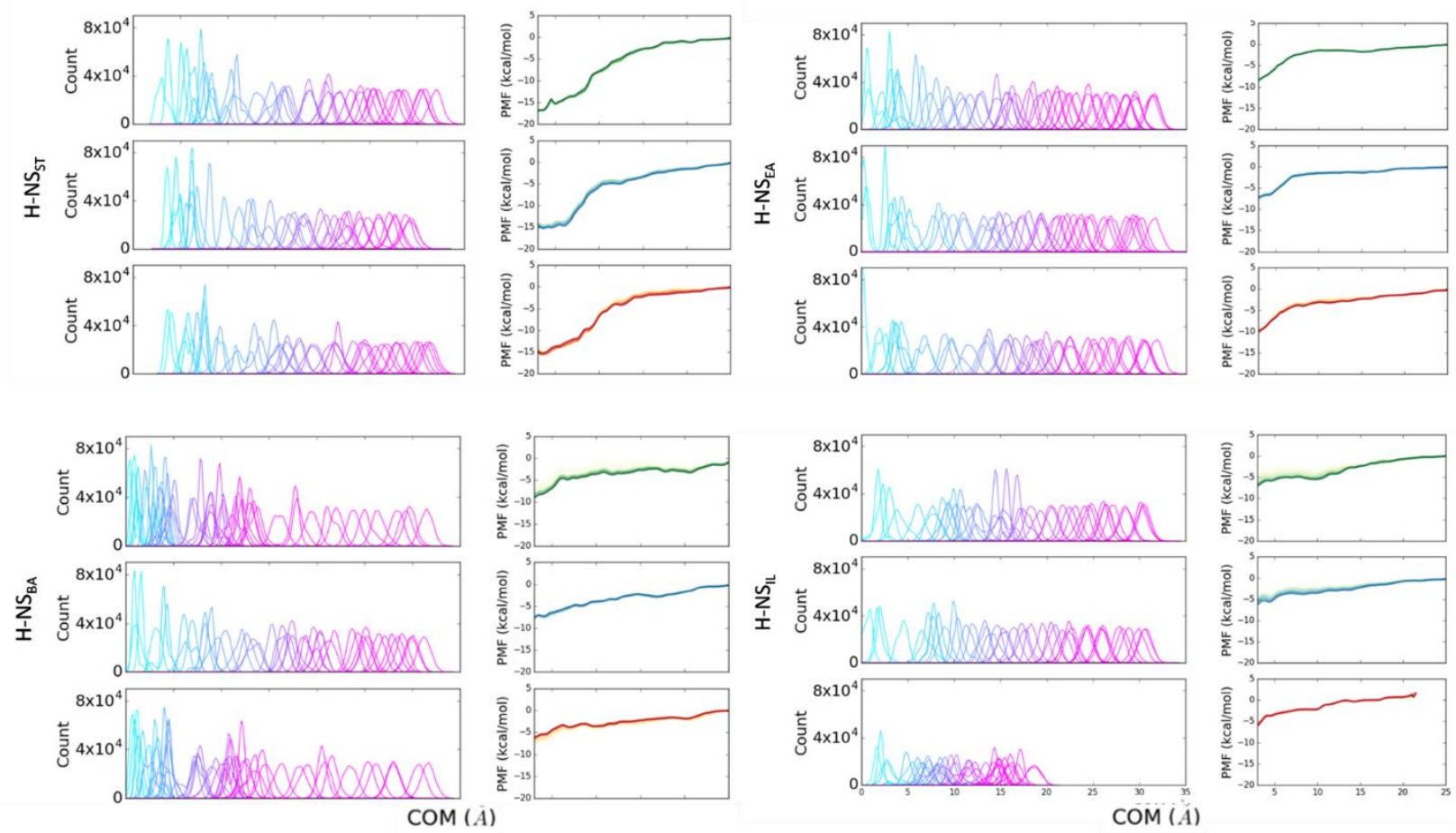

$\operatorname{COM}(A)$

Figure 3 - Figure Supplement S3. The umbrella histogram and convergency to simulate the $\mathrm{H}$-NS orthologs site2 dimer at different simulation conditions: $293 \mathrm{~K}, 0.15 \mathrm{M} \mathrm{NaCl}$ (Green), 293 $\mathrm{K}, 0.50 \mathrm{M} \mathrm{NaCl}$ (Blue), and $313 \mathrm{~K}, 0.15 \mathrm{M} \mathrm{NaCl}$ (Red). Each PMF subplot contains a series of 5 curves from 38 to 54 ns/window (4 ns increment), demonstrating convergence. 


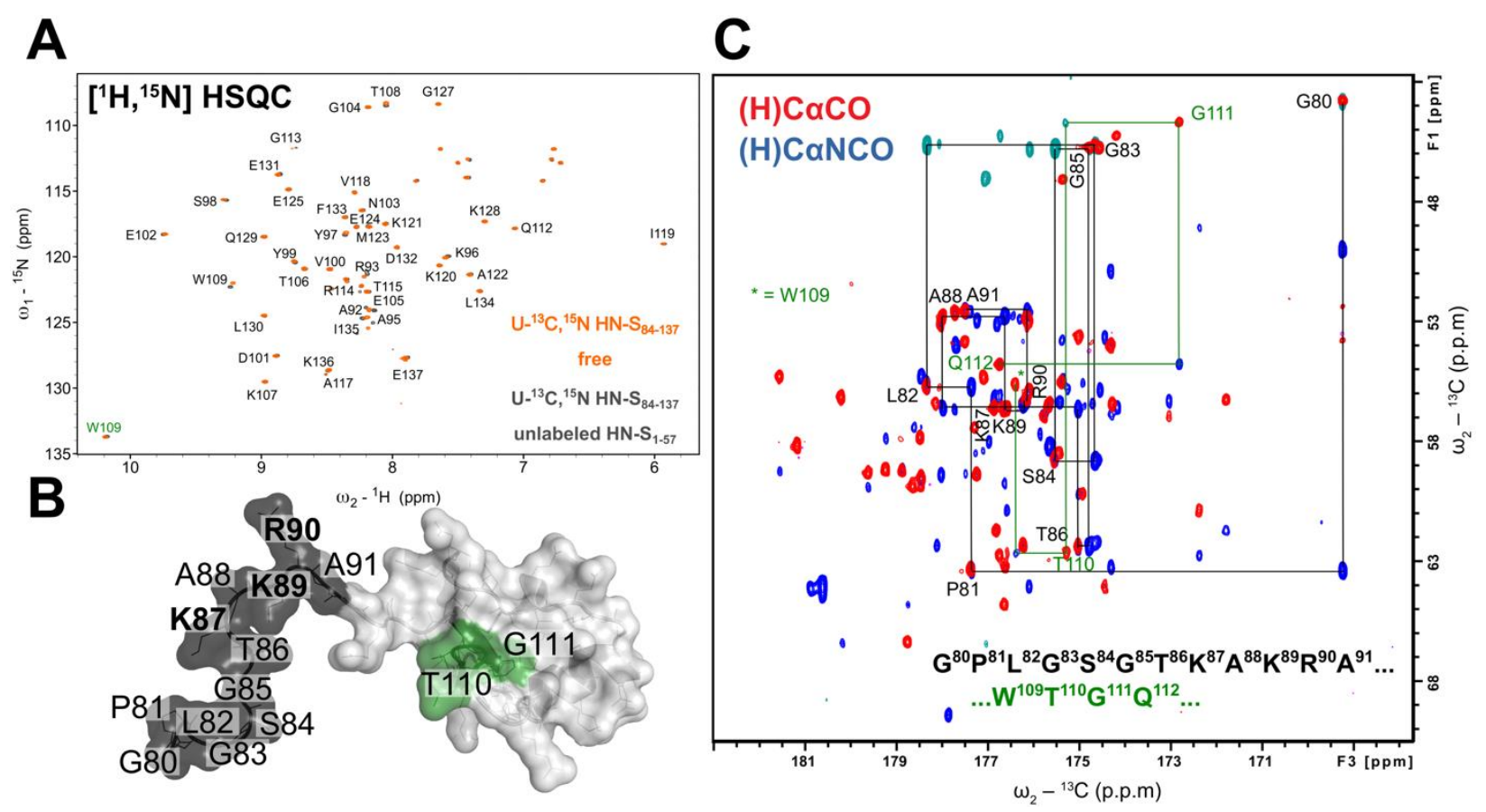

Figure 4 - Figure Supplement S4. The low- $y{ }^{13} \mathrm{C}$-detected experiments unveil the molecular details of highly dynamic and solvent exposed residues elusive for classical ${ }^{1} \mathrm{H}$-detected approaches. The panel A shows the ${ }^{1} \mathrm{H}-{ }^{15} \mathrm{~N}$ HSQC spectrum of $200 \mathrm{uM}{ }^{13} \mathrm{C},{ }^{15} \mathrm{~N}$-labelled $S$. typhimurium $\mathrm{H}-\mathrm{NS}_{84-137}$ protein in $\mathrm{Apo}$ (orange) and $\mathrm{H}-\mathrm{NS}_{1-57}$ saturated $(1.5 \mathrm{mM}$ ) form (dark gray). The residues that are missing from $\mathrm{H} / \mathrm{N}$ correlation ${ }^{1} \mathrm{H}$-detected experiments are depicted in panel $\mathbf{B}$ with black and green mapped on the NMR solution structure of $\mathrm{H}-\mathrm{NS}_{91-137}$ (PDB id $1 \mathrm{HNR}$; the lowest energy structure). The flexible residues of S84-A91 were added together with GPLG artificial residues left after the tag cleavage. The only tryptophan side chain $\mathrm{H} \varepsilon / \mathrm{N} \varepsilon$ imidazole correlation is marked W109 in green. The $\mathbf{C}$ panel presents the overlay of 2D $(\mathrm{H}) \mathrm{CACO}$ and $(\mathrm{H}) \mathrm{CANCO}{ }^{13} \mathrm{C}$-detected experiments and sequential walk for two regions, $\mathrm{G}^{80}$ $A^{91}$ marked black and $T^{110}-G^{111}$ marked green, that are not detected on $\mathbf{A}$. A complete sequential carbon walk can be done for the entire protein $\mathrm{H}_{-} \mathrm{NS}_{84-137}$ sequence. 